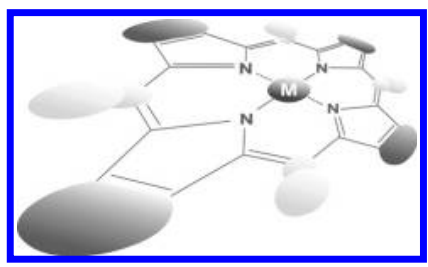

\title{
Glycosylated porphyrin-cucurbituril conjugate for photodynamic inactivation of bacteria and doxorubicin carriage for anticancer drug delivery
}

\author{
Melis Özkan ${ }^{a}$, Yağmur Keser ${ }^{a}$, Ahmet Koc $^{b}$ and Dönüs Tuncel ${ }^{\star a, b}$ \\ anstitute of Materials Science and Nanotechnology, National Nanotechnology Research Center (UNAM), \\ Bilkent University, Ankara, 06800, Turkey \\ ${ }^{\mathrm{b}}$ Department of Chemistry, Bilkent University, 06800 Ankara, Turkey
}

This paper is part of the 2019 Women in Porphyrin Science special issue.

Received 12 September 2019

Accepted 2 October 2019

\begin{abstract}
Porphyrin derivatives are highly attractive in the construction of multifunctional molecular platforms with interesting properties and applications. In this regard, we report here the use of a multifunctional porphyrin-based molecular platform as a photosensitizer for photodynamic therapy and as a drug carrier. This molecular platform was constructed by conjugating a host molecule, cucurbit[7]uril to a triglycosylated tetraphenyl porphyrin and serves very efficiently as a photosensitizer in the inactivation of both gram-negative (Escherichia coli, E. coli) and gram-positive bacteria (Bacillus subtilis, B. subtilis) and growth inhibition of cancer cells as well as a doxorubicin (DOX) carrier for chemo-photodynamic dual cancer therapy. Another remarkable feature of this photosensitizer is that it shows negligible cytotoxicity in the dark.
\end{abstract}

KEYWORDS: porphyrin, cucurbituril, multi-functional molecular platform, light-activated antimicrobial agent, photodynamic effect, doxorubicin delivery, anticancer therapy.

\section{INTRODUCTION}

Porphyrin-based photosensitizers continue to attract great attention because a photoactive porphyrin core can be conveniently substituted with a variety of functional groups in order to prepare a photosensitizer with desirable properties [1, 2]. Photosensitizers have a wide range of applications including photodynamic therapy for the inactivation of bacteria and cancerous tumors [3-7]. In order to bring out the photodynamic effect, mainly three components are required which are photosensitizer, molecular oxygen and a suitable light source [8-10]. Molecular oxygen is converted into highly reactive oxygen species (ROS) when the photosensitizer is irradiated with light. Singlet oxygen, among other ROS, is highly toxic and kills the nearby cells efficiently.

*Correspondence to: Dönüs Tuncel, tel.: (90)312 2902420, email: dtuncel@fen.bilkent.edu.tr.
Ideally it is desirable for photosensitizers to be watersoluble and show no toxicity in the absence of a light source [10].

Another advantage of porphyrin-based photosensitizers is that by attaching ionic or hydrophilic groups their water-solubility can be enhanced [11, 12]. Furthermore, by decorating their periphery with bulky substituents, $\pi-\pi$ interactions between porphyrin cores and, as a result aggregate formation, can be reduced and singlet oxygen generation efficiency can be increased $[11,12]$. Recently, a supramolecular approach started to emerge as an attractive way to design an ideal photosensitizer because using this approach it would be possible to regulate the photosensitizer's cytotoxicity, solubility and optical properties [13-18]. In this regard, we reported a highly water-soluble supramolecular photosensitizer based on [5]rotaxane which was prepared by a 1,3-dipolar cycloaddition reaction between the alkyne substituted porphyrin core and azide functionalized stopper groups 
in the presence of cucurbit[6]uril (CB6) [19]. This rotaxane-based photosensitizer shows negligible toxicity in the dark and has been proven to be a very efficient photosensitizer for photodynamic bacteria inactivation and cancer therapy.

Recently we have also prepared a multifunctional molecular platform by conjugating a host molecule, cucurbit[7]uril (CB7) to a trimannosylated tetraphenyl porphyrin (TPP) core through copper-catalyzed azidealkyne cycloaddition (CuAAC) reaction (Chart 1) and demonstrated its potentiality as a photosensitizer owing to its ability to generate singlet oxygen in high yield [23]. In this paper, by taking advantage of this feature we explored its application in photodynamic antibacterial and cancer therapy as well as its capability as a drug carrier due to the presence of the CB7 host.

\section{EXPERIMENTAL}

Synthesis and singlet oxygen $\left({ }^{1} \mathrm{O}_{2}\right)$ quantum yield determination of TPP-3Man-CB7 was reported in our previous publication [20].

\section{Bacterial culture preparation}

A single colony of E. coli (DH5-Alpha) and B. subtilis (MTCC 441) on a semi-solid Lysogeny Broth (LB) agar plate was placed into $5.0 \mathrm{ml}$ liquid LBs and incubated in the dark for $16 \mathrm{~h}$ at $37^{\circ} \mathrm{C}, 200 \mathrm{rpm}$. Then, centrifugation for $2 \mathrm{~min}$ at $4{ }^{\circ} \mathrm{C}, 7000 \mathrm{rpm}$ was applied to the harvest bacteria. They were washed three times by fresh $\mathrm{pH} 7.4$ (1X) Gibco phosphate-buffered saline (PBS) (purchased from Thermo Fisher Scientific). The supernatant was decanted and the residue was resuspended in PBS and then diluted to $\sim 10^{8} \mathrm{CFU} / \mathrm{ml}$.

\section{Evaluation of the effect of TPP-3Man-CB7 on bacterial growth kinetics}

Initially, the suspensions of bacteria $\left(\sim 10^{6} \mathrm{CFU} / \mathrm{ml}\right)$ were tested in a 96-well plate. The bacterial solution was kept in the dark for 45 min to reach the exponential growth phase. 12 concentrations of TPP-3Man-CB7 were prepared in PBS as follows: 0.49, 0.98, 1.46, 1.94, $2.41,2.88,3.34,3.80,4.26,4.71,5.15$ and $5.60 \mu \mathrm{M}$. Then, TPP-3Man-CB7 solutions were incubated with bacterial culture at $37^{\circ} \mathrm{C}$ for $15 \mathrm{~min}$. 96-well plates were exposed to white light for $20 \mathrm{~s}, 40 \mathrm{~s}$ and $1 \mathrm{~min}\left(20 \mathrm{~mW} / \mathrm{cm}^{2}\right)$. The absorbance of each set was measured at $600 \mathrm{~nm}$ during the time periods of $1 \mathrm{~h}, 2 \mathrm{~h}, 4 \mathrm{~h}, 8 \mathrm{~h}, 12 \mathrm{~h}, 16 \mathrm{~h}$ and $24 \mathrm{~h}$ (SpectraMax M5 multi-detection microplate reader system). Control samples were prepared using equal volumes of PBS. The experiments were performed in triplicate. The same protocol was followed in the dark as well. The effect of TPP-3Man-CB7 on the growth kinetics of B. subtilis was also studied using the same experimental procedure.

\section{Evaluation of photodynamic biocidal efficacy of TPP-3Man-CB7 against E. coli and B. subtilis}

$2.0 \mathrm{ml}$ portions of prepared E. coli suspensions $\left(\sim 10^{8}\right.$ $\mathrm{CFU} / \mathrm{ml}$ ) were incubated with 3.34 and $5.60 \mu \mathrm{M}$ of TPP3Man-CB7 in the dark for $15 \mathrm{~min}$ at $37^{\circ} \mathrm{C}, 200 \mathrm{rpm}$ and then exposed to white light for $1 \mathrm{~min}\left(20 \mathrm{~mW} / \mathrm{cm}^{2}\right)$. After that, the bacterial mixtures were serially diluted $10^{5}$-fold with PBS. $50 \mu 1$ volumes from each bacterial mixture were spread on the semi-solid LB agar plates and placed into an incubator $\left(37^{\circ} \mathrm{C}\right)$ for $16 \mathrm{~h}$. The same experiment was performed in the dark as well. For the control sets, equal volumes of PBS were used. The similar experimental protocol was used for $B$. subtilis.

\section{SEM analysis of $E$. coli-TPP-3Man-CB7 interaction}

Similar method reported in our previous publication was adopted [22].

\section{Cytotoxicity and photocytotoxicity assay}

Cell culture. MCF7 cells were cultured in DMEM supplemented with $10 \%$ heated-inactivate FBS, containing $1 \%$ penicillin and streptomycin at $37^{\circ} \mathrm{C}$ and $5 \% \mathrm{CO}_{2}$-humidified atmosphere. When the cells reached 80-90\% confluency, they were washed twice by PBS $(\mathrm{pH}=7.4)$ and detached with Trypsin EDTA $(0.25 \%)$. The cells were centrifuged at $1300 \mathrm{rpm}$ for $5 \mathrm{~min}$, and then they were re-plated onto a T75 flask at an appropriate density based on each experimental scale.

MTT cell viability assay. In vitro dark- and photocytotoxicity of TPP-3Man-CB7, anti-cancer activities of Doxorubicin (DOX) and the combined anti-proliferative and dark- and photo-cytotoxicity activities of TPP3Man-CB7 + DOX on MCF7 cells were determined by the MTT cell viability assay. To determine the dark cytotoxicity, the cells were seeded onto 96-well plates at a density of $4 \times 10^{3}$ cells/well, incubated overnight, and then the cells were treated with various concentrations of TPP-3Man-CB7 $(1-100 \mu \mathrm{M})$, DOX $(2-60 \mu \mathrm{M})$ and TPP-3Man-CB7 + DOX complex (1-100 $\mu$ M TPP-3Man-CB7 + 2-60 $\mu$ M DOX) for $96 \mathrm{~h}$. To make the TPP-3Man-CB7 + DOX complex, DOX and TPP-3Man-CB7 were stirred together at certain concentrations of each for $30 \mathrm{~min}$ at $600 \mathrm{rpm}$ at $37^{\circ} \mathrm{C}$. To determine the photo-cytotoxicity, irradiation treatment was performed with white light $\left(20 \mathrm{~mW} / \mathrm{cm}^{2}\right)$ for $5 \mathrm{~min}$, $72 \mathrm{~h}$ after of the cells were seeded, and then the cells were placed in the dark for another $24 \mathrm{~h}$ at $37^{\circ} \mathrm{C}$. All dilution series of treatment groups and untreated control samples included an equal volume of PBS and DMEM was prepared for each treatment study (Table S1). All different treatments were carried out in triplicate. For the MTT assay, thiazolyl blue tetrazolium bromide (MTT), a membrane-permeable dye (ab146345) was used according to the manufacturer's protocol. The medium was removed and the cells were incubated with $110 \mu \mathrm{L}$ 
of DMEM containing [3-(4,5-dimethylthiazol-2-yl)-2,5diphenyltetrazolium bromide] (MTT, $1.2 \mathrm{mM}$ ) for $4 \mathrm{~h}$ at $37^{\circ} \mathrm{C}$. Then, $110 \mu \mathrm{L}$ of SDS-HCl solution $(1 \mathrm{~g}$ SDS in $10 \mathrm{~mL}$ of $10 \mathrm{mM} \mathrm{HCl}$ ) was added to each well. The plates were incubated for a further $16-18 \mathrm{~h}$ at $37^{\circ} \mathrm{C}$, and the cell viability of each well was measured at a wavelength of $570 \mathrm{~nm}$ using a microplate reader.

Statistical analysis. A similar method to that reported in our previous publication was adopted [22].

\section{RESULTS AND DISCUSSION}

\section{Synthesis and singlet oxygen generation capacity of TPP-3Man-CB7}

The multifunctional molecular platform, abbreviated as TPP-3Man-CB7 and shown in the Chart 1, has been synthesized and characterized as reported in our previous publication [23]. This molecular platform contains a photoactive tetraphenyl porphyrin (TPP) core that will allow singlet oxygen generation. The TPP core is decorated with three hydrophilic mannosyl groups to increase water solubility and efficient interaction with bacteria cell walls and cell membranes. The advantage of mannose is to provide good targeting ability toward bacteria [24]. CB7 is installed on one arm of the TPP as a receptor for an additional drug delivery and provides bulkiness to reduce interactions between the TPP units. Recently, we have also prepared glucosylated porphyrin-thiophene-based nanoparticles and shown their antibacterial effect against gram-negative and grampositive strains [25].

Singlet oxygen generation efficiency of TPP3Man-CB7 was determined as around $80 \%$ using a singlet oxygen trap molecule, 1,3-diphenylisobenzofuran (DPBF) as reported in our previous publication [23]. Shortly, time-dependent decreases in the absorbance of DPBF under light exposure and in the presence of photosensitizer were recorded and correlated with the singlet oxygen generation efficiency. This result suggested that TPP-3Man-CB7 can be used as an efficient photosensitizer in photodynamic therapy. By taking advantage of this feature, we studied next its lightactivated antibacterial efficacy.

Investigation of TPP-3Man-CB7 effect on growth kinetics of gram-negative bacteria, Escherichia coli $(E$. coli), and gram-positive bacteria, Bacillus subtilis (B. subtilis)

The use of photodynamic effects in the inactivation of bacteria has been receiving considerable attention because of the antibiotic resistance of bacteria $[21,22]$. In this context, we first investigated the effect of TPP-3Man-CB7 on growth kinetics of $E$. coli (DH5-Alpha strain), gramnegative bacteria, and B. subtilis (MTCC 441 strain), gram-positive bacteria. Growth kinetic studies of both strains were performed using 12 different concentrations of TPP-3Man-CB7 varying from 0.49 to $5.60 \mu \mathrm{M}$ in the dark and upon white light irradiation $\left(20 \mathrm{~mW} / \mathrm{cm}^{2}\right)$ for $20 \mathrm{~s}, 40 \mathrm{~s}$ and $1 \mathrm{~min}$ separately (Figs S1-S8). While TPP-3Man-CB7 displays negligible toxicity even at high concentrations in the dark, it has a dose-dependent effect on bacterial growth when irradiated with white light. Moreover, the light exposure time plays an important role. For $B$. subtilis, at $5.60 \mu \mathrm{M}$, the highest concentration, there is a considerable difference between $20 \mathrm{~s}, 40 \mathrm{~s}$ and 1 min exposure (Fig. 1). Two crucial parameters, TPP3Man-CB7 concentration and light exposure time, were optimized and the ideal condition was found to be $5.60 \mu \mathrm{M}$ TPP-3Man-CB7 concentration and 1 min light exposure for both strains (Figs 1 and 2).

\section{Investigation of cytotoxicity and phototoxicity against E.coli}

To visualize the dose-dependent effect of TPP3Man-CB7, we further conducted bacterial survival

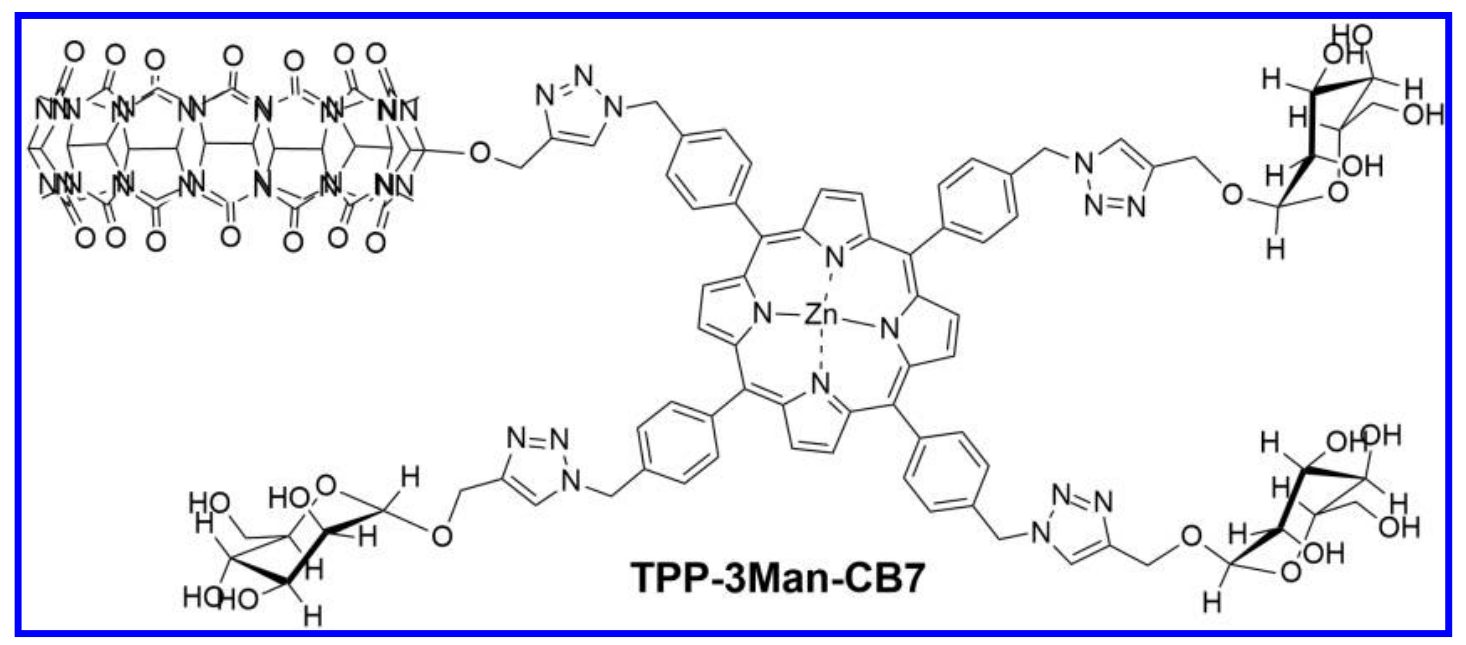

Chart 1. Molecular structure of the multifunctional molecular platform 


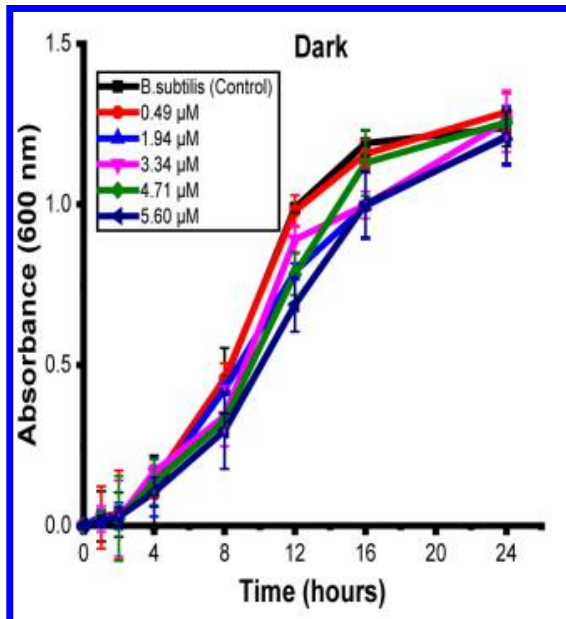

(a)

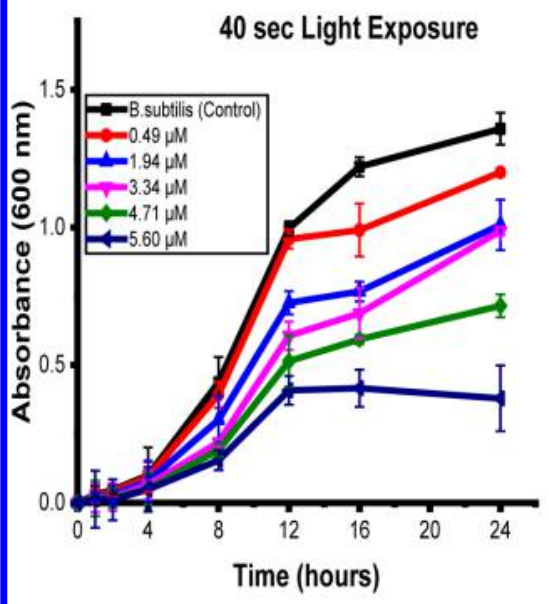

(c)

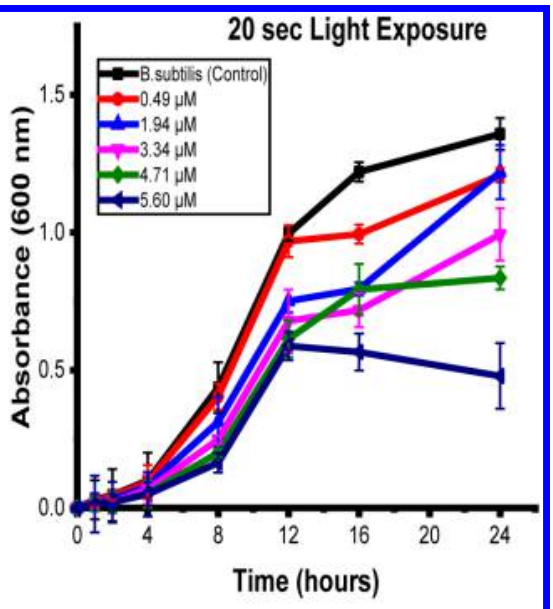

(b)

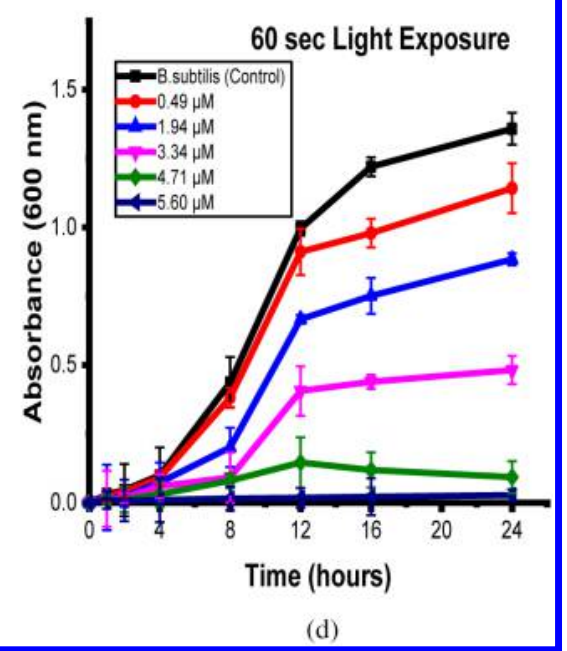

Fig. 1. Growth kinetic studies of $B$. subtilis (a) in the dark (b) upon white light irradiation $\left(20 \mathrm{~mW} / \mathrm{cm}^{2}\right)$ with the exposure time of $20 \mathrm{~s}$ (c) $40 \mathrm{~s}$ (d) $1 \mathrm{~min}$. All results are demonstrated as mean $\pm \operatorname{SD}(n=3)$

experiments using various concentrations $(2.41,3.80,4.71$ and $5.60 \mu \mathrm{M}$ ) of TPP-3Man-CB7 in the dark (Figs 3a, 3b) and upon exposure to white light for $1 \mathrm{~min}$ (as optimum according to growth kinetic studies) by a surface plating method (Figs 4a-4e). Colony counting results confirmed that the inhibition efficiency upon irradiation of $E$. coli suspension incubated with $2.41 \mu \mathrm{M}$ was $47 \%, 3.80 \mu \mathrm{M}$ 90\%, $4.71 \mu \mathrm{M} 98 \%$ and $5.6 \mu \mathrm{M}$ TPP-3Man-CB7 100\%, while in the dark it is only around $10 \%$.

\section{Investigation of interactions between}

TPP-3Man-CB7 and $E$. coli under scanning electron microscopy (SEM)

The interaction of E. coli with TPP-3Man-CB7 was also analyzed under scanning electron microscopy (SEM). To this end, E. coli suspensions were treated with $5.60 \mu \mathrm{M}$ TPP-3Man-CB7 in the dark and under light. In Figs $5 \mathrm{a}$ and $5 \mathrm{~b}$, the SEM images of E. coli without any treatment and $5.60 \mu \mathrm{M}$ TPP-3Man-CB7-treated were respectively shown in the dark conditions. In the dark, rod-like shape of $E$. coli were preserved without any damage. Upon light treatment and incubation with TPP-3Man-CB7, the morphology of E. coli was completely changed (Fig. 5d) in comparison with its light treated control set (Fig. 5c). As can be seen, the bacteria are destroyed and amalgamated as debris, clearly indicating the high efficiency of TPP-3Man-CB7 as a photosensitizer.

\section{Investigation of cytotoxicity, photocytotoxicity on MCF7 cell line and doxorubicin carriage efficiency}

To investigate the effect of TPP-3Man-CB7 on cancer therapy, in vitro dark- and photo-cytotoxicity of TPP3Man-CB7 was tested on the MCF7 breast cancer cell line through the MTT cell viability assay. MCF7 cells were treated with various concentrations of TPP-3Man-CB7 


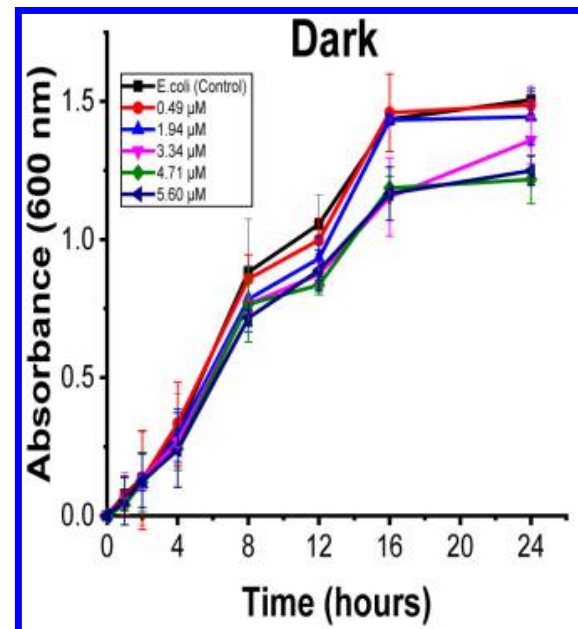

(a)

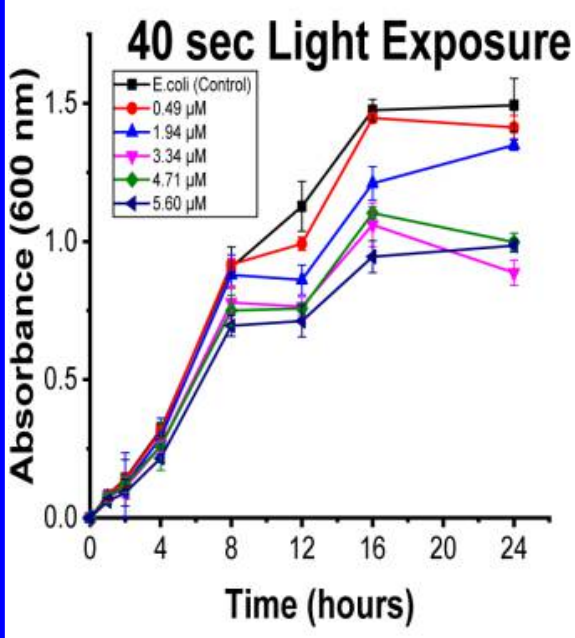

(c)

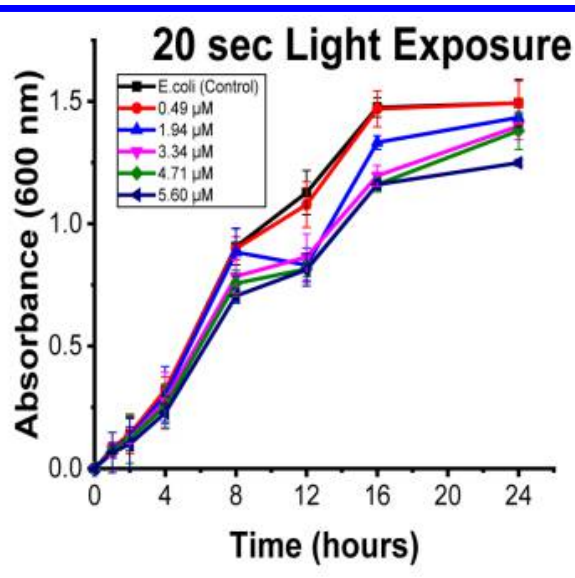

(b)

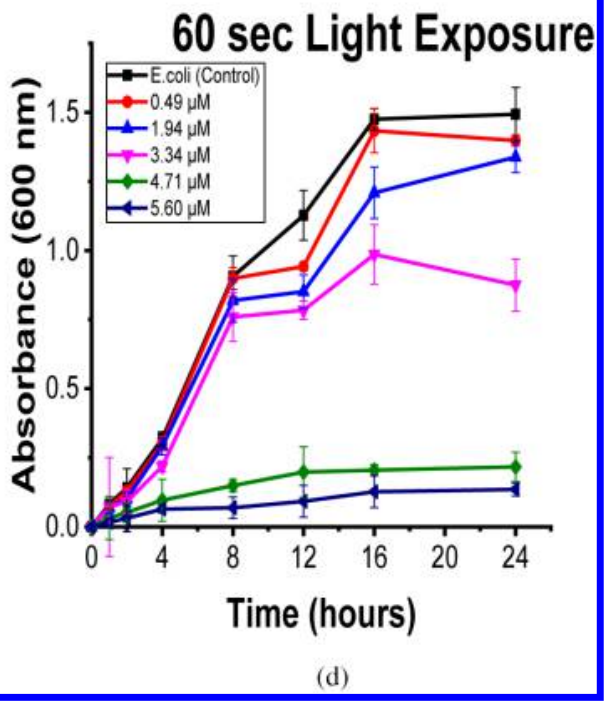

Fig. 2. Growth kinetic studies of E. coli (a) in the dark (b) upon white light irradiation $\left(20 \mathrm{~mW} / \mathrm{cm}^{2}\right)$ with the exposure time of $20 \mathrm{~s}$ (c) $40 \mathrm{~s} \mathrm{(d)} 1 \mathrm{~min}$. All results are demonstrated as mean $\pm \mathrm{SD}(n=3)$

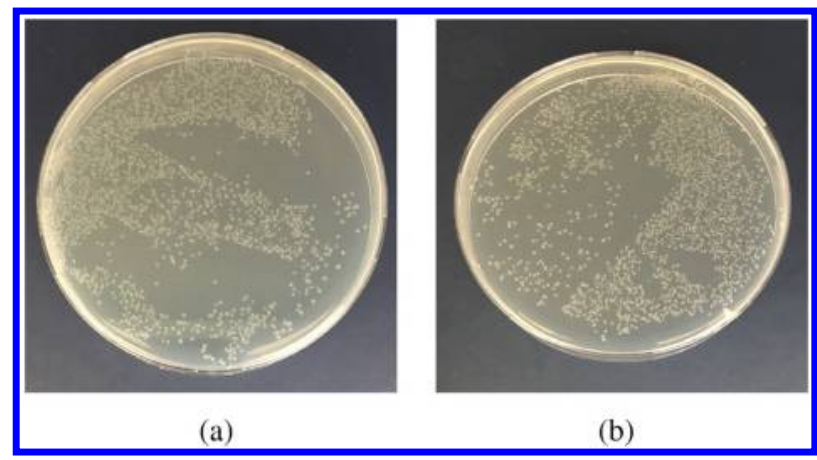

Fig. 3. Plate photographs for E. coli on semi-solid LB agar plate (a) without TPP-3Man-CB7 in the dark, (b) $5.60 \mu \mathrm{M}$ TPP3Man-CB7-treated in the dark

(1-100 $\mu \mathrm{M}$ ) together with a DMEM control group to assess the dark cytotoxicity (Table S1, Supporting information). To investigate its photo-cytotoxicity, cells were exposed to white light $\left(20 \mathrm{~mW} / \mathrm{cm}^{2}\right)$ for $5 \mathrm{~min}$. Not surprisingly, the results confirmed that cells treated with TPP-3Man-CB7 in dark had a high cell survival rate and it is validated that TPP-3Man-CB7 did not show any cytotoxic effect under dark environment (Fig. 6a).

In contrast, cells treated with TPP-3Man-CB7 and exposed to light showed a dramatic decrease in cell viability; in addition, by increasing concentration of TPP-3Man-CB7, the inhibition rate increased gradually. While cells treated with TPP-3Man-CB7 in dark had a half maximal inhibitory concentration value $\left(\mathrm{IC}_{50}\right)$ of $>100 \mu \mathrm{M}$, this value was decreased at around $35 \mu \mathrm{M}$ after PDT. Cell viability dropped at around $80 \%$ and $5 \%$ for the lowest $(5 \mu \mathrm{M})$ and highest $(100 \mu \mathrm{M})$ concentrations of TPP-3Man-CB7, respectively, compared to the DMEM control group $(p<0.0001)$ (Fig. 6b). These results clearly show that even at low concentrations, white light activates TPP-3Man-CB7 efficiently, causing a significant decrease in cell viability. The results indicate 


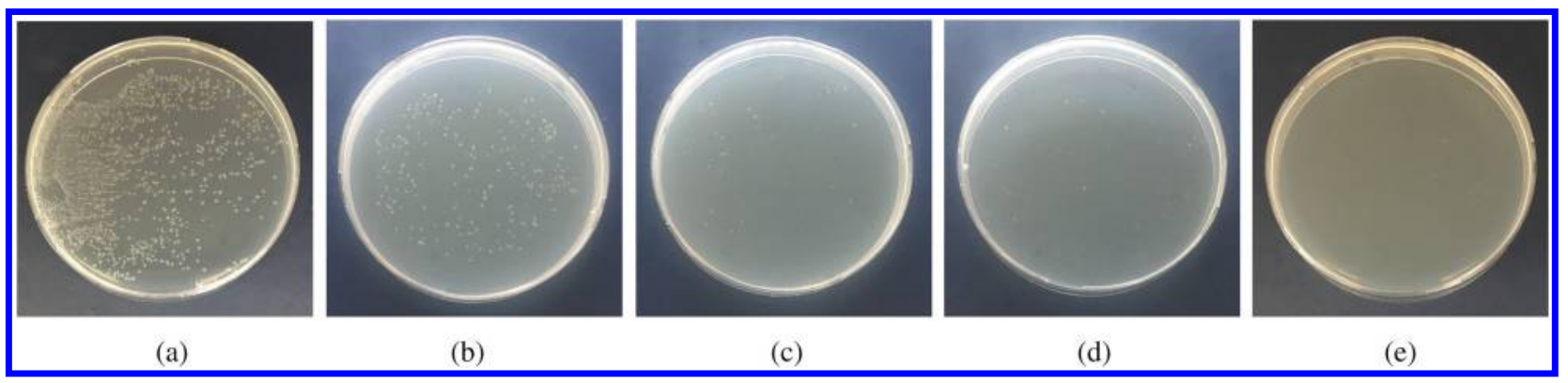

Fig. 4. Plate photographs for E. coli on semi-solid LB agar plates under photo-irradiation (a) without TPP-3Man-CB7 (b) $2.41 \mu \mathrm{M}$ TPP-3Man-CB7-treated (c) $3.80 \mu \mathrm{M}$ TPP-3Man-CB7-treated (d) $4.71 \mu \mathrm{M}$ TPP-3Man-CB7-treated (e) $5.60 \mu \mathrm{M}$ TPP-3Man-CB7-treated

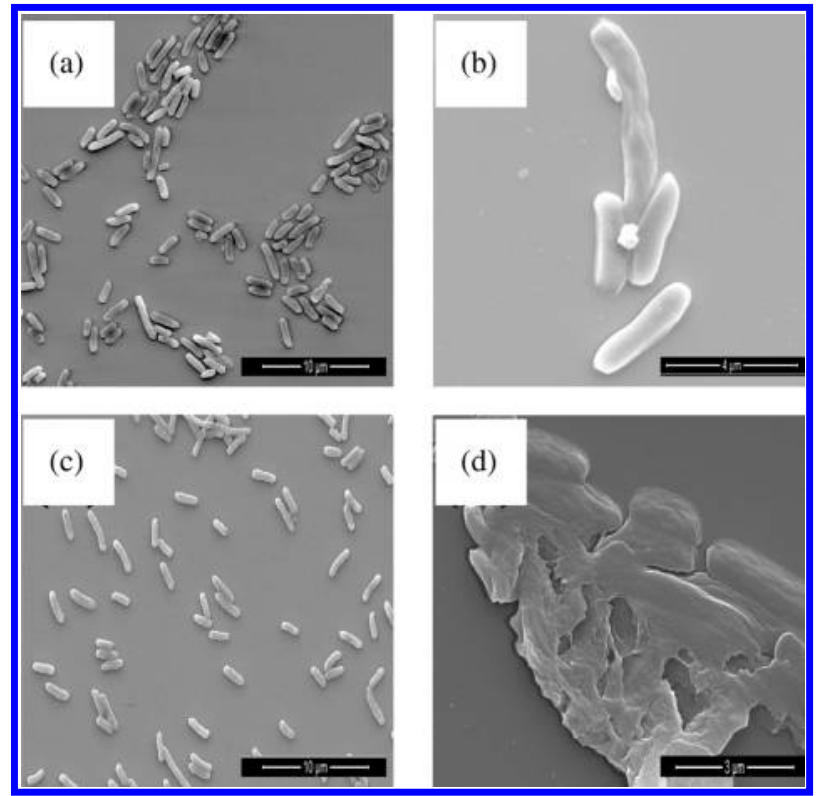

Fig. 5. SEM Analysis of E. coli-TPP-3Man-CB7 Interaction. (a) E. coli. only, dark control, (b) $5.60 \mu \mathrm{M}$ TPP-3Man-CB7treated in the dark, (c) E. coli. only, light control $\left(20 \mathrm{~mW} / \mathrm{cm}^{2}\right.$ white light, $1 \mathrm{~min}$ exposure), (d) $5.60 \mu \mathrm{M}$ TPP-3Man-CB7treated under white light $\left(20 \mathrm{~mW} / \mathrm{cm}^{2}\right.$ white light, $1 \mathrm{~min}$ exposure)

that TPP-3Man-CB7 is a very efficient photosensitizer exhibiting negligible cytotoxicity in the dark but high toxicity upon brief exposure to white light with low fluency.

As our molecular platform contains host molecule (CB7) which that is available for host-guest chemistry, the next logical strategy was to employ this molecular platform as a drug carrier to enhance the toxicities and targeted delivery efficiencies of chemotherapeutics. Here, a chemotherapy drug, doxorubicin (DOX) [26] was selected as a well-known modal chemotherapeutic molecule because it inhibits the growth of tumorigenic cells by blocking an topoisomerase II in order to inhibit macromolecular biosynthesis by suppressing transcription [23]; it can also form complexes with CB7 and CB8, which makes it a good candidate as a drug to be carried by TPP-3Man-CB7 [26-30].

Therefore, we investigated the drug carrier capability of TPP-3Man-CB7 to find out whether this molecular platform can be employed for a dual chemo and photodynamic therapy [31-33]; TPP-3Man-CB7 + DOX complexes were prepared at certain concentrations of DOX and TPP-3Man-CB7 in DMEM. An MTT cell viability assay was employed to evaluate the toxicity of TPP-3Man-CB7 + DOX complex on the MCF7 breast cancer cell line. Cells were incubated for $96 \mathrm{~h}$ with different concentrations of TPP-3Man-CB7 + DOX complex in dark and under light irradiation $(5 \mathrm{~min}$, $20 \mathrm{~mW} / \mathrm{cm}^{2}$ ). As a control, MCF7 cells were treated with various concentrations of DOX and exhibited a dosedependent decrease in cell viability; we ensured that light did not affect the toxicity of DOX (Figs 6a, 6b). It can be seen that DOX inhibits the cell viability dramatically even at low concentrations in dark when carried with TPP-3Man-CB7, compared to that of DOX and TPP-3Man-CB7 alone. This might be due to strong interactions of DOX molecules with $\mathrm{CB}$, which results in a slow release. This particular point can be useful if slow and sustained releases of drugs are targeted.

However, the inhibition on cell viability was further enhanced when the cells were treated with TPP3Man-CB7 + DOX complex and exposed to light. The cell viability was about $1 \%$ at the concentration of $65 \mu \mathrm{M}$ TPP-3Man-CB7 +35 $\mu \mathrm{M}$ DOX and decreased almost $100 \%$ at the highest concentration $(100 \mu \mathrm{M}$ TPP-3Man-CB7 +60 $\mu$ M DOX) by PDT; while this high inhibition rate was not achieved under light when DOX and TPP-3Man-CB7 were used individually (Fig. 6b). Moreover, it is also clearly seen that the complex decreased the cell viability more under light than that of the comparison in dark, which offers the use of these complexes in small concentrations in order to achieve high toxicity, thanks to photodynamic feature of TPP-3Man-CB7, eliminating side effects of chemotherapeutics. These results clearly support the 


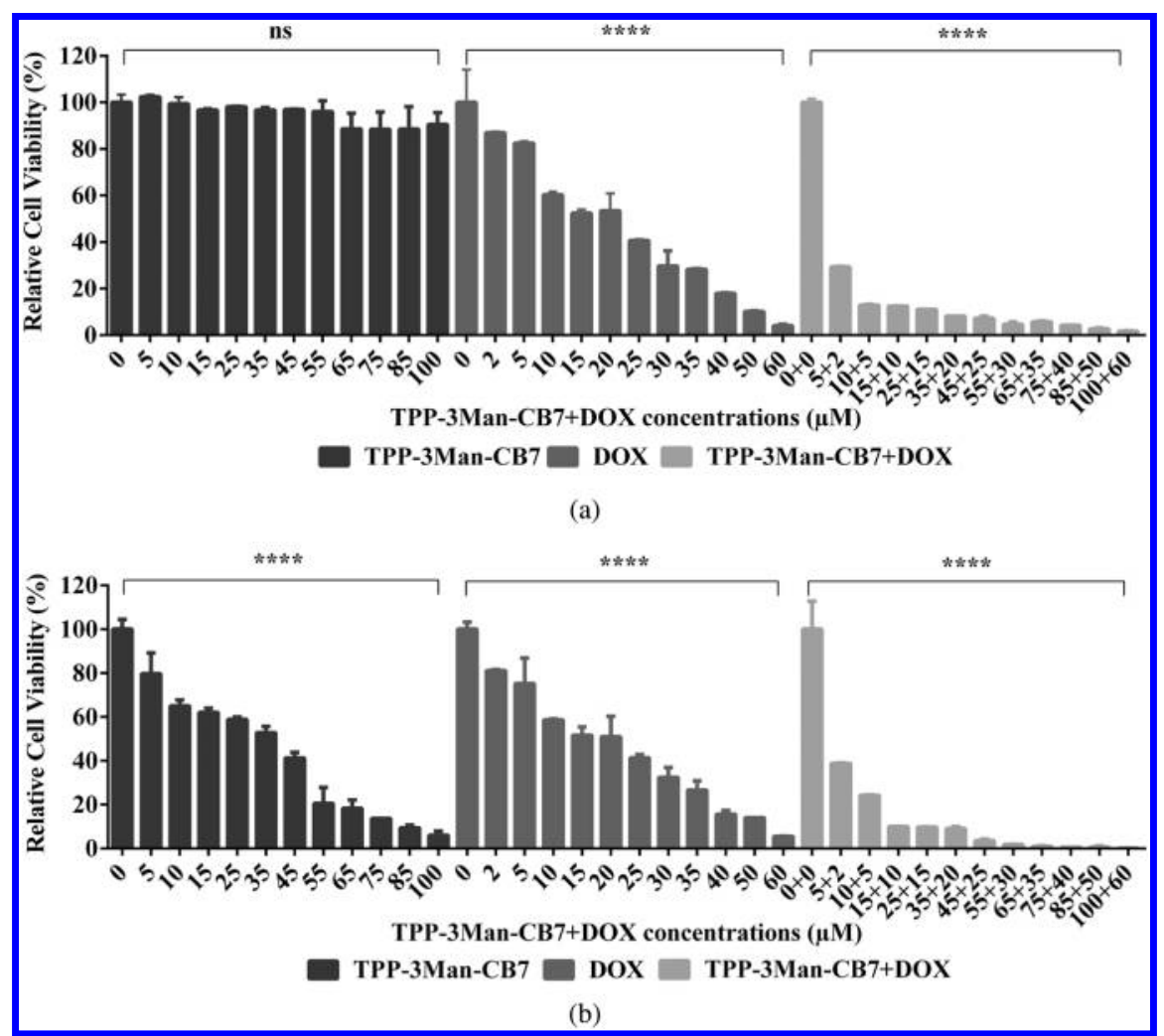

Fig. 6. In vitro relative cell viability (\%) measurements using MCF-7 breast cancer cell line through a MTT assay at the given concentrations $(\mu \mathrm{M})$ of TPP-3Man-CB7, DOX and TPP-3Man-CB7 + DOX (a) in dark, (b) upon white light irradiation (5 min, $20 \mathrm{~mW} / \mathrm{cm}^{2}$ ) treatments along with DMEM control group in MCF7 cells. Differences in MTT relative cell viability (normalized to DMEM control group) were analyzed for each dose separately using one-way ANOVAs followed by multiple comparisons (Tukey's at $\alpha=0.05$; Graphpad). $* * * * p<0.0001$; ns: non-significant

synergistic chemo and photodynamic effects suggesting that TPP-3Man-CB7 can be utilized conveniently both as a photosensitizer and as a drug carrier for a dual chemo/photo therapy.

\section{CONCLUSION}

In summary, a multifunctional molecular platform constructed through $\mathrm{Cu}$-catalyzed click reaction of triglycosylated azide containing porphyrin core with monopropargyloxy-CB7 has been employed as a photosensitizer for antibacterial and cancer phototherapy. This photosensitizer shows negligible cytotoxicity against both gram-positive and gram-negative bacteria as well as MCF-7 cancer cells even at high doses but becomes highly toxic under even low doses of white light, killing almost $100 \%$ of bacteria and cells tested.

It was also demonstrated that this molecular platform can be used as a drug carrier and shows high potentiality as an agent for a dual photo/chemotherapy.

\section{Acknowledgments}

We acknowledge the financial support of The Scientific and Technological Research Council of Turkey-TÜBİTAK (KBAG 215Z035).

\section{Supporting information}

Growth kinetic studies and cell viability assay concentrations (Figs S1-S8 and Table S1) are given in the supplementary material. This material is available free of charge via the Internet at http://www.worldscinet. com/jpp/jpp.shtml.

\section{REFERENCES}

1. Beletskaya I, Tyurin VS, Tsivadze AY, Guilard R and Stern C. Chem. Rev. 2009; 109: 1659-1713.

2. Drain CM, Varotto A and Radivojevic I. Chem. Rev. 2009; 109: 1630-1658.

-3. Moret F and Reddi E. J. Porphyrins Phthalocyanines 2017; 21: 239-256.

4. Singh S, Aggarwal A, Bhupathiraju NVSDK, Arianna G, Tiwari K and Drain CM. Chem. Rev. 2015; 115: 10261-10306.

-5. Sternberg ED, Dolphin D and Brückner C. Tetrahedron 1998; 54: 4151-4202.

-6. Ethirajan M, Chen Y, Joshi P and Pandey RK. Chem. Soc. Rev. 2011; 40: 340-362.

7. Rajora MA, Lou JWH and Zheng G. Chem. Soc. Rev. 2017; 46: 6433-6469. 
-8. Phillips D. Photochem. Photobiol. Sci. 2010; 9: 1589-1596.

-9. Wilson BC and Patterson MS. Phys. Med. Biol. 2008; 53: R61-R109.

10. Zhou Z, Song J, Nie L and Chen X. Chem. Soc. Rev. 2016; 45: 6597-6626.

11. Xu L, Liu L, Liu F, Li W, Chen R, Gao Y and Zhang W. J. Mater. Chem. B, 2015; 3: 3062-3077.

12. Ma D, Liu ZH, Zheng QQ, Zhou XY, Zhang Y, Shi YF, Lin JT and Xue W. Macromol. Rapid Commun. 2013; 34: 548-552.

13. Li X, Lee S and Yoon J. Chem. Soc. Rev. 2018; 47: 1174-1188.

14. Lei W, Jiang G, Zhou Q, Hou Y, Zhang B, Cheng X and Wang X. Chem. Phys. Chem. 2013; 14: 1003-1008.

15. Liu K, Liu Y, Yao Y, Yuan H, Wang S, Wang Z and Zhang X. Angew. Chem., Int. Ed. 2013; 52: 8285-8289.

16. Chen L, Bai H, Xu JF, Wang S and Zhang X. ACS Appl. Mater. Interfaces. 2017; 9: 13950-13957.

17. Lei W, Jiang G, Zhou Q, Zhang B and Wang X. Phys. Chem. Chem. Phys. 2010; 12: 13255-13260.

18. Caceres J, Robinson-Duggon J, Tapia A, Paiva C, Gomez M, Bohne C and Fuentealba D. Phys. Chem. Chem. Phys. 2017; 19: 2574-2582.

19. Özkan M, Keser Y, Hadi SE and Tuncel D. Eur. J. Org. Chem. 2019; 3534-3541.

20. Koc A, Khan R and Tuncel D. Chem. -Eur. J. 2018; 24: 15550-15555.
21. Maisch T. Photochem. Photobiol. Sci. 2015; 14: $1518-1526$.

22. Maisch T. Mini. Rev. Med. Chem. 2009; 974-998.

-23. Tacar O, Sriamornsak P and Dass CR. J. Pharm. Pharmacol. 2013; 65: 157-170.

-24. Liu M, Li J and Li B. Langmuir 2018; 34: 1574-1580.

-25. Khan R, Özkan M, Khaligh A and Tuncel D. Photochem. Photobiol. Sci. 2019; 18: 1147-1155.

-26. Wu X, Zhang YM and Liu Y. RSC Adv. 2016; 6: 99729-99734.

-27. Benyettou F, Fahs H, Elkharrag R, Bilbeisi RA, Asma B, Rezgui R, Motte L, Magzoub M, Brandel J, Olsen JC, Piano F, Gunsalus KC, Platas-Iglesias C and Trabolsi A. RSC Adv. 2017; 7: 23827-23834.

28. Macartney DH. Isr. J. Chem. 2011; 51: 600-615.

29. Walker S, Oun R, McInnes FJ and Wheate NJ. Isr. J. Chem. 2011; 51: 616-624.

30. Park KM, Suh K, Jung H, Lee DW, Ahn Y, Kim J, Baek K and Kim K. Chem. Commun. 2009; 71-73.

31. Kralova J, Kejik Z, Briza T, Pouckova P, Kral A, Martasek P and Kral V. J. Med. Chem. 2010; 53: 128-138.

32. Lim WQ, Yang G, Zeng S, Phua F, Chen H and Zhao Y. ACS Appl. Mater. Interfaces. 2019; 11: 16391-16401.

33. Zhang W, Li Y, Sun JH, Tan CP, Ji LN and Mao ZW. Chem. Commun. 2015; 51: 1807-1810. 